\title{
A New Global Asymptotic Stability Result of Delayed Neural Networks via Nonsmooth Analysis
}

\author{
Yaning Gu, Deyou Liu, Wenjuan Wu, Jingwen Zhang \\ College of Science, Yanshan University, Qinhuangdao, China \\ E-mail: \{wwkgogps919, liudeyouysu\}@163.com \\ Received December 28, 2009; revised January 29, 2010; accepted February 26, 2010
}

\begin{abstract}
In the paper, we obtain new sufficient conditions ensuring existence, uniqueness, and asymptotic stability of the equilibrium point for delayed neural network via nonsmooth analysis, which makes use of the Lipschitz property of the functions. Based on this tool of nonsmooth analysis, we first obtain a couple of general results concerning the existence and uniqueness of the equilibrium point. Then we drive some new sufficient conditions ensuring global asymptotic stability of the equilibrium point. Finally, there are the illustrative examples feasibility and effectiveness of our results. Throughout our paper, the activation function is a more general function which has a wide application.
\end{abstract}

Keywords: Delayed Neural Networks, Global Asymptotic Stability, Nonsmooth Analysis

\section{Introduction}

In recent years, the stability of a unique equilibrium point of delayed neural networks has extensively been discussed by many researchers [1-5]. Several criteria ensuring the global asymptotic stability of the equilibrium point are given by using the comparison method, Lyapunov functional method, M-matrix, diagonal dominance technique and linear matrix inequality approach. In [1-5], some sufficient conditions are given for the global asymptotic stability of delayed neural networks by constructing Lyapunov functions. A new sufficient condition on the global asymptotic stability for delayed neural networks via nonsmoosh analysis is derived in this letter. The condition is independent of delay and imposes constraints on both the feedback matrix and delayed feedback matrix. Our results generalize and improve the preciously known works due to expending the activation function of the delay part.

Concerning the global stability of delayed neural networks described by the following differential equations with time delays

$$
\dot{x}=-g(x(t))+A f(x(t))+A^{\tau} h(x(t-\tau))+u
$$

where $x(t)=\left(x_{1}(t), \cdots, x_{n}(t)\right)^{T} \in \mathfrak{R}^{n}$

$$
g(x(t))=\left(g_{1}\left(x_{1}(t)\right), \cdots, g_{n}\left(x_{n}(t)\right)\right)^{T} \in \mathfrak{R}^{n}
$$

$$
\begin{aligned}
& f(x(t))=\left(f_{1}\left(x_{1}(t)\right), \cdots, f_{n}\left(x_{n}(t)\right)\right)^{T} \in \mathfrak{R}^{n} \\
& h\left(x(t-\tau)=\left(h_{1}\left(x_{1}(t-\tau), \cdots, h_{n}\left(x_{n}(t-\tau)\right)\right) \in \mathfrak{R}^{n}\right.\right.
\end{aligned}
$$

and $A=\left(a_{i j}\right), \quad A^{\tau}=\left(a_{i j}^{\tau}\right) \in \mathfrak{R}^{n \times n}$ are respectively, the feedback matrix and the delayed feedback matrix. $u=$ $\left(u_{1}, u_{2}, \cdots, u_{n}\right)^{T} \in \mathfrak{R}^{n}$ is a constant input vector and $\tau$ is the delay parameter. Furthermore, we assume that the function $g$, the activation function $f$ and the activation function $h$ satisfy the following conditions:

A1) Each function $g_{i}: \mathfrak{R} \rightarrow \mathfrak{R}$ is a locally Lipschitz function and there exists $m_{i}>0$ such that $g_{i}^{\prime}(y) \geq m_{i}$ for all at $y \in \mathfrak{R}$ which $g_{i}$ is differentiable.

A2) Each function $f_{i}: \Re \rightarrow \Re$ is a globally Lipschitz function with module $k_{i}>0$, i.e.,

$$
\begin{aligned}
&\left|f_{i}\left(y_{1}\right)-f_{i}\left(y_{2}\right)\right| \leq k_{i}\left|y_{1}-y_{2}\right| \\
& \forall i=1, \cdots, n \text { and } y_{1}, y_{2} \in \mathfrak{R}
\end{aligned}
$$

A3) Each function $h_{i}: \mathfrak{R} \rightarrow \mathfrak{R}$ is a globally Lipschitz function with module $l_{i}>0$, i.e.,

$$
\begin{array}{r}
\left|h_{i}\left(y_{1}\right)-h_{i}\left(y_{2}\right)\right| \leq l_{i}\left|f_{i}\left(y_{1}\right)-f_{i}\left(y_{2}\right)\right| \\
\forall i=1, \cdots, n \text { and } y_{1}, y_{2} \in \Re
\end{array}
$$


The paper is organized as follows: Section 2 contains a short introduction to nonsmooth analysis for Lipschitz functions. In particular, the Lipschitzin Hadamard Theorem is explained and a homeomorphism theorem is obtained. Section 3 is to demonstrate how nonsmooth analysis can be carried out on (1) to derive sufficient conditions to ensure the existence and uniqueness of the equilibrium point of (1). In Section 4, we study new sufficient conditions with guarantee the GAS of the (1). In Section 5, we have an illustrative example and its simulations. We conclude in Section 6.

Notation: Let $m_{i}, k_{i}, l_{i}, i=1,2, \cdots, n$ be the constants given in assumptions (A1), (A2) and (A3), define two diagonal matrices $M=\operatorname{diag}\left(m_{1}, m_{2}, \cdots, m_{n}\right)$ and $K$ $=\operatorname{diag}\left(k_{1}, k_{2}, \cdots, k_{n}\right)$

Let $m=\min _{i}\left\{m_{i}\right\}, \quad k=\max _{i}\left\{k_{i}\right\}$ and $l=\max _{i}\left\{l_{i}\right\}$.

Let $\|\cdot\|$ denotes the Euclidean norm for vectors and the matrix norm for matrices for any vector $v=\left(v_{1}, v_{2}\right.$, $\left.\cdots, v_{n}\right) \in \mathfrak{R}^{n} \quad|v|=\left(\left|v_{1}\right|,\left|v_{2}\right|, \cdots,\left|v_{n}\right|\right)^{T}$ similarly, for any matrix $B=\left(b_{i j}\right) \in \mathfrak{R}^{n \times n},|B|=\left(\left|b_{i j}\right|\right)$.

Let $\rho(B)$ denote the spectral radius of $B$. It is known that $\rho(B) \leq \rho(|B|)$. Moreover,

$$
\rho(B) \geq \rho(\tilde{B}) \text { if } B \geq \tilde{B} \geq 0 .
$$

$B$ is called a $P$ matrix ( $P_{0}$ matrix ) if and only if all principal minors of $B$ are positive (nonnegative) and denoted by $B \in P\left(B \in P_{0}\right)$. For any matrix $B \in \mathfrak{R}^{n \times n}, \quad \mu_{2}(B)=(1 / 2) \lambda_{\text {max }}\left(B+B^{T}\right)$ i.e., $\mu_{2}(B)$ is the largest eigenvalue of the symmetric part of $B$. All the mathematical facts concerning the eigenvalues of a matrix used in this paper can be found in the book [6].

\section{Nonsmooth Analysis on Lipschitz Functions}

We first review some concepts which are essential for conducting nonsmooth analysis on Lipschitz function. Then we state the Lipschitzian Hadamard Theorem, which gives conditions for homeomorphism of Lipschitz functions. Finally, we give a sufficient condition which ensures the existence and uniqueness of the equilibrium point of (1) for any input vector $u \in \mathfrak{R}^{n}$.

Let the function $F: \mathfrak{R}^{n} \rightarrow \mathfrak{R}^{n}$ be locally Lipschitzian.

According to Rademacher's theorem [7], $F$ is differentiable almost everywhere. Let $D_{F}$ denote the set of those points where $F$ is differentiable and $F^{\prime}(x)$ denote the Jacobian of $F$ at $x \in D_{F}$. For any given $x \in \mathfrak{R}^{n}$, define the constant

$$
\operatorname{Lip}_{x} F:=\sup _{\substack{y \rightarrow x \\ x \neq y \in \mathfrak{R}^{n}}} \frac{\|F(y)-F(x)\|}{\|y-x\|}
$$

Since $F$ is locally Lipschitz, the constant $\operatorname{Lip}_{x}$ is finite and we have

$$
\left\|F^{\prime}(x)\right\| \leq \operatorname{Lip}_{x} F \quad \text { for any } x \in D_{F} .
$$

Now we are ready to define the generalized Jacobian in the sense of Clarke [8]:

For any $x \in \mathfrak{R}^{n}$, let $\partial F$ be the set of the following collection of matrices

$$
\begin{aligned}
& \partial F(x)=c o\{W \mid \text { there exists a sequence of } \\
& \left.\qquad\left\{x^{k}\right\} \subset D_{F} \text { with } \lim _{x^{k} \rightarrow x} F^{\prime}\left(x^{k}\right)=W\right\}
\end{aligned}
$$

where $\operatorname{co} \Omega$ denotes the convex hull of the set $\Omega$. It is easy to see that the above definition is well defined and $\|W\| \leq \operatorname{Lip}_{x} F$ for any $W \in \partial F(x)$.We say that $\partial F(x)$ is invertible if every element $W$ in $\partial F$ is nonsingular.

For any given $x, y \in \mathfrak{R}^{n}$, the Lebourg Theorem [8] states that there exists an element $W$ in the union $U_{z \in[x, y]} \partial F(z)$ such that

$$
F(y)-F(x)=W(y-x)
$$

where $[x, y]$ denotes the segment connecting $x$ and $y$.

For any two locally Lipschitz functions $F: \mathfrak{R}^{n} \rightarrow \mathfrak{R}^{n}$, $\rightarrow \mathfrak{R}^{n}, \quad G: \mathfrak{R}^{n} \rightarrow \mathfrak{R}^{n}$, we have

$$
\partial(F+G) \subseteq \partial F(x)+\partial G(x) \text { for all } x \in \mathfrak{R}^{n} .
$$

Now we are ready to state the Lipschitzian Hadamard Theroem which will lead to our homeomorphism result Theorem 1.

Lemma 1 [9] (Lipschitzian Hadamard Theorem): Suppose $F: \mathfrak{R}^{n} \rightarrow \mathfrak{R}^{n}$, is locally Lipschitzian and let $\kappa>0$. If $\partial F(x)$ is invertible and $\left\|W^{-1}\right\| \leq \kappa$ for all $x \in \mathfrak{R}^{n}$ and all $W \in \partial F(x)$, then $F$ is a homeomorphism from $\mathfrak{R}^{n}$ onto $\mathfrak{R}^{n}$.

For more discussions on the generalized Jacobian and its various applications, please refer to books $[7,8]$ as well as to the paper $[10,11]$. Now, we analyze (1) from the viewpoint of nonsmooth analysis. We first recall that a state $x^{*} \in \Re^{n}$ is called an equilibrium point of (1) if it satisfies

$$
-g\left(x^{*}\right)+A f\left(x^{*}\right)+A^{\tau} h\left(x^{*}\right)+u=0
$$

To study the existence and uniqueness of the equilibrium point for any input vector $u \in \mathfrak{R}^{n}$, we define the function $F: \mathfrak{R}^{n} \rightarrow \mathfrak{R}^{n}$, by

$$
F(x)=g(x)-A f(x)-A^{\tau} h(x)-u
$$


Naturally, $F$ is a locally Lipschitz function since $g, f$ and $h$ are so. Moreover, the generallized Jacobian of $F$ at $x$ is overestimated by (3). If each of the activation function $f_{i}, h_{i}$ is nondecreasing on the real line $\Re$, then we have a more accurate estimate of $\partial F(x)$ as follows (see (4)).

$$
\begin{gathered}
\partial F(x) \subseteq \tilde{V}=\left\{\begin{array}{c}
W \in \mathfrak{R}^{n \times n} \mid=C-A D-l A^{\tau} D, \\
C=\operatorname{diag}\left(c_{1}, c_{2}, \cdots, c_{n}\right), \\
D=\operatorname{diag}\left(d_{1}, d_{2}, \cdots, d_{n}\right) \\
\text { with } c_{i} \geq m_{i},-k_{i} \leq d_{i} \leq k_{i}, \\
\text { for all } i=1,2, \cdots, n\}
\end{array}\right\} \\
\partial F(x) \subseteq \tilde{V}=\left\{\begin{array}{r}
W \in \mathfrak{R}^{n \times n} \mid=C-A D-l A^{\tau} D, \\
C=\operatorname{diag}\left(c_{1}, c_{2}, \cdots, c_{n}\right), \\
D=\operatorname{diag}\left(d_{1}, d_{2}, \cdots, d_{n}\right) \\
\text { with } c_{i} \geq m_{i}, 0 \leq d_{i} \leq k_{i}, \\
\text { for all } i=1,2, \cdots, n\}
\end{array}\right\}
\end{gathered}
$$

Clearly, $\tilde{V} \subset V$. By applying Theorem 1 to (3) and (4), we have the following.

Theorem 1: Suppose functions $f, g$ and $h$ satisfy assumptions (A1), (A2) and (A3) and that one of the following two conditions holds.

1) Each element $W \in V$ is nonsingular.

2) Each element $W \in \tilde{V}$ is nonsingular and each activation function $f_{i}$ and $h_{i}$ is nondecreasing.

Then for each input vector $u \in \mathfrak{R}^{n}$, the function $F$ in (2) is a homeorphism from $\mathfrak{R}^{n}$ onto $\mathfrak{R}^{n}$.

Proof: We only prove the result for the case (i). The case (2) be proved similarly as (1).

To show that $F$ is a homeomorphism from $\mathfrak{R}^{n}$ to $\mathfrak{R}^{n}$, in accordance with Theorem 1 it suffices to prove that the norms of inverses of all elements in $V$ are uniformly bounded. In other words, we need to show that there exists a positive constant $\kappa$ such that

$$
\left\|W^{-1}\right\| \leq \kappa \quad \text { for all } \quad W \in V
$$

We prove it by a contradiction. Assume that there exists a sequence of matrices $\left\{W^{k}\right\} \subset V$ satisfying

$$
\left\|W^{-1}\right\| \rightarrow \infty
$$

Then there exist two sequences of diagonal matrices $\left\{C^{k}\right\}$ and $\left\{D^{k}\right\}, C^{k}=\operatorname{diag}\left(c_{1}^{k}, c_{2}^{k}, \cdots, c_{n}^{k}\right), D^{k}=$ $\operatorname{diag}\left(d_{1}^{k}, d_{2}^{k}, \cdots, d_{n}^{k}\right)$ with $c_{i}^{k} \geq m_{i}$ and $d_{i}^{k} \in\left[-k_{i}, k_{i}\right]$ for all $i=1, \cdots, n$ such that

$$
W^{k}=C^{k}-A D^{k}-l A^{\tau} D^{k}
$$

Recall that $M=\operatorname{diag}\left(m_{1}, m_{2}, \cdots, m_{n}\right)$ and let $P^{k}=$ $M^{-1} C^{k}=\operatorname{diag}\left(p_{1}^{k}, p_{2}^{k}, \cdots, p_{n}^{k}\right)$.It is easy to see that $p_{i}^{k} \geq 1$ for all $i=1, \cdots, n$

$$
\left\|\left(P^{k}\right)^{-1}\right\| \leq 1
$$

and $W^{k}=\left(M-A D^{k}\left(P^{k}\right)^{-1}-l A^{\tau} D^{k}\left(P^{k}\right)^{-1}\right) P^{k}$.

Since both sequences of diagonal matrices $\left\{D^{k}\right\}$ and $\left\{\left(P^{k}\right)^{-1}\right\}$ are bounded, without loss of generality, we assume that

$$
\lim _{k \rightarrow \infty} D^{k}\left(P^{k}\right)^{-1}=Q=\operatorname{diag}\left(q_{1}, q_{2}, \ldots, q_{n}\right)
$$

For some $q_{k} \in\left[-k_{i}, k_{i}\right], \quad i=1, \cdots, n$. We observe that the matrix sequence

$$
\left\{M-A D^{k}\left(P^{k}\right)^{-1}-l A^{\tau} D^{k}\left(P^{k}\right)^{-1}\right\}
$$

and the matrix $\left\{M-A Q-l A^{\tau} Q\right\}$ all belong to the collection $V$. Hence, those matrices are nonsingular. Moreover, for all $k$ sufficiently large, we have

$$
\begin{aligned}
\left\|\left(W^{k}\right)^{-1}\right\| & \leq\left\|\left(P^{k}\right)^{-1}\right\| . \\
& \left\|\left(M-A D^{k}\left(P^{k}\right)^{-1}-l A^{\tau} D^{k}\left(P^{k}\right)^{-1}\right)^{-1}\right\| \\
& \leq 2\left\|\left(M-A Q-l A^{\tau} Q\right)^{-1}\right\|
\end{aligned}
$$

This contradicts our assumption (6). Hence, there exists a constant $\kappa>0$ such that (5) holds. It then follows from Theorem 1 that $F$ is a Homeomorphism from $\Re^{n}$ onto itself.

The above homeomorphism result means that if each element in $V$ is nonsingular, then the neural network defined by (1) has a unique equilibrium point for any input vector $u \in \mathfrak{R}^{n}$. This result is the starting point of the next two sections where we will consider what practical conditions make the delay neural network stable.

\section{Existence and Uniqueness of the Equilibrium Point}

In this section, based on Theorem 1 we present some new sufficient conditions which ensure the existence and uniqueness of the equilibrium point of (1). As consequences, we further show that the existence assumption on equilibrium point is unnecessary in some existing results for GAS.

Theorem 2: Suppose one of the following assumptions holds:

1) $\rho\left(\left|A+l A^{\tau}\right| K M^{-1}\right)<1$;

2) $-\left(A+l A^{\tau}\right) \in P_{0}$ and each activation function is nondecreasing; 
3) $\mu_{2}\left(A+l A^{\tau}\right)<m / k$ and each activation function is nondecreasing;

Then for each input vector $u \in \mathfrak{R}^{n}$, the function $F(\cdot)$ is a homeomorphism from $\mathfrak{R}^{n}$ onto $\mathfrak{R}^{n}$.

Proof: 1) we first recall the facts that for any matrix $B \in \mathfrak{R}^{n \times n}, \quad \rho(B) \leq \rho(|B|)$. And that if two nonnegative matrices $B, \tilde{B} \in \mathfrak{R}^{n \times n}$ satisfy $B \leq \tilde{B}$, then we have $\rho(B) \leq \rho(B)$. It follows from Theorem 1 that we need only to show that each element in $\mathrm{W}$ is nonsingular.

Let $W$ be any element in $V$, then there exist two diagonal matrices $C=\operatorname{diag}\left(c_{1}, c_{2}, \cdots, c_{n}\right)$ and $D$ $=\operatorname{diag}\left(d_{1}, d_{2}, \cdots, d_{n}\right)$ with $c_{i} \geq m_{i}$ and $d_{i} \in\left[-k_{i}, k_{i}\right]$ for all $i=1,2, \cdots, n$ such that

$$
W=C-A D-l A^{\tau} D
$$

We have from the assumption i) of the theorem and the mathematical facts listed at the beginning of the proof that

$$
\begin{aligned}
\rho\left(\left(A+l A^{\tau}\right) D C^{-1}\right) & \leq \rho\left(\left(\left|A+l A^{\tau}\right|\right)|D| C^{-1}\right) \\
& \leq \rho\left(\left(A+l A^{\tau}\right) K M^{-1}\right)<1
\end{aligned}
$$

This means that the matrix $I-\left(A+l A^{\tau}\right) D C^{-1}$ is nonsingular. Then the nonsingularity of $W$ follows from the observation that

$$
W^{-1}=C^{-1}\left(I-\left(A+l A^{\tau}\right) D C^{-1}\right)^{-1}
$$

For the remaining two cases, we need only to show that each element in $V$ is nonsingular. We note the fact $d_{i} \geq 0$ for all $i=1, \ldots, n$ in defining $\tilde{V}$.

2) The proof is trivial by noticing the fact that for any three matrices $B_{1}, B_{2}, Q$, in $\Re^{n \times n}$ with $B_{1}$ being positive diagonal, $B_{2} \in P_{0}$ and $Q$ a nonnegative diagonal matrix, then the matrix $B_{1}+B_{2} Q \in P$, hence it is nonsingular. Any matrix we encountered in $W$ has the same structure as of $B_{1}+B_{2} Q$ and therefore it is nonsingular.

3) As in 2), it suffices to show that any element $W$ in $V$ is nonsingular. Then there exist two diagonal matrices $C=\operatorname{diag}\left(c_{1}, c_{2}, \cdots, c_{n}\right), \quad D=\operatorname{diag}\left(d_{1}, d_{2}, \cdots, d_{n}\right) \quad$ with $c_{i} \geq m_{i}$ and $d_{i} \in\left[0, k_{i}\right]$ for all $i=1, \cdots, n$ such that $W=C-A D-l A^{\tau} D$. Since $C$ is nonsingular, it suffices to show the matrix $N=I-\left(A+l A^{\tau}\right) D C^{-1}$ is nonsingular. We prove it by a contradiction. Assume that $N$ is singular, then there exists $0 \neq x \in \mathfrak{R}^{n}$ such that $N x=0$, or equivalently

$$
\left(\frac{m}{k}\right)\left(A+l A^{\tau}\right) D C^{-1} x=\left(\frac{m}{k}\right) x
$$

Let $Q=(m / k) D C^{-1}=\operatorname{diag}\left(q_{1}, q_{2}, \cdots, q_{n}\right)$, then $0<q_{i}<1$, for all $i=1,2, \cdots, n$.

Multiplying $(Q x)^{T}$ on both sides of (7), we have

$$
(Q x)^{T}\left(A+l A^{\tau}\right) Q x=\left(\frac{m}{k}\right) x^{T} Q x
$$

which yields that

$$
\begin{aligned}
\frac{m}{k}\|Q x\|^{2}= & \left(\frac{m}{k}\right) x^{T} Q^{2} x \leq\left(\frac{m}{k}\right) x^{T} Q x \\
& \leq \mu_{2}\left(A+l A^{\tau}\right)\|Q x\|^{2}
\end{aligned}
$$

That is

$$
\left(\frac{m}{k}-\mu_{2}\left(A+l A^{\tau}\right)\right)\|Q x\|^{2} \leq 0
$$

Since $Q x \neq 0$ (otherwise it would follow from (7) that $x=0$ ), we have

$$
\frac{m}{k} \leq \mu_{2}\left(A+l A^{\tau}\right)
$$

a contradiction to the assumption $\mu_{2}\left(A+l A^{\tau}\right)<m / k$.

Hence any element $W \in \tilde{V}$ is nonsingular. This completes our proof.

\section{New Conditions for GAS}

In this section, we present new conditions for the GAS of the equilibrium point of (1). We assume that all the activation function are nondecreasing, i.e., the generalized Jacobian of $F$ at any point $x \in \mathfrak{R}^{n}$ is contained in $\tilde{V}$.

Theorem 3: In addition to assumptions (A1), (A2) and (A3), we assume that each activation function is nondecreasing. Suppose

$$
2 \mu_{2}\left(A+l A^{\tau}\right)+\left\|I A^{\tau}-I\right\|^{2}<2\left(\frac{m}{k}\right)
$$

Then for each $u \in \mathfrak{R}^{n}$, (1) has a unique equilibrium point which is GAS.

In particular, the DCNN where $m=k=1$ has a unique equilibrium point for each input vector $u \in \mathfrak{R}^{n}$ and this equilibrium point is GAS if the following condition holds:

$$
2 \mu_{2}\left(A+l A^{\tau}\right)+\left\|l A^{\tau}-I\right\|^{2}<2
$$

Proof: Since $2 \mu_{2}\left(A+l A^{\tau}\right)+\left\|l A^{\tau}-I\right\|^{2}<2(m / k)$, we obviously have $\mu_{2}\left(A+l A^{\tau}\right)<(m / k)$. Then it follows from Theorem 2 3) that (1) has a unique equilibrium 
point. Hence it remains to show that this equilibrium point, say $x^{*}$, is GAS. For simplicity, we shift $x^{*}$ to this origin through the transformation

$$
\begin{aligned}
& z(t)=x(t)-x^{*} \\
& z(t-\tau)=x(t-\tau)-x^{*}
\end{aligned}
$$

Equation (1) then can be equivalently written as the following system

$$
\begin{aligned}
\dot{z}(t)=- & \left(g\left(z(t)+x^{*}\right)-g\left(x^{*}\right)\right) \\
& +A \Phi(z(t))+A^{\tau} \Psi(z(t-\tau))
\end{aligned}
$$

where

$$
\begin{aligned}
& z(\cdot)=\left(z_{1}(\cdot), z_{2}(\cdot), \cdots, z_{n}(\cdot)\right)^{T} \\
& \Phi(z(\cdot))=\left(\phi_{1}\left(z_{1}(\cdot)\right), \phi_{2}\left(z_{2}(\cdot)\right), \cdots, \phi_{n}\left(z_{n}(\cdot)\right)\right)^{T} \\
& \Psi(z(\cdot))=\left(\varphi_{1}\left(z_{1}(\cdot)\right), \varphi_{2}\left(z_{2}(\cdot)\right), \cdots, \varphi_{n}\left(z_{n}(\cdot)\right)\right)^{T}
\end{aligned}
$$

and

$$
\begin{aligned}
& \phi_{i}\left(z_{i}(\cdot)\right)=f_{i}\left(z_{i}(\cdot)+x^{*}\right)-f_{i}\left(x^{*}\right) \\
& \varphi_{i}\left(z_{i}(\cdot)\right)=h_{i}\left(z_{i}(\cdot)+x^{*}\right)-h_{i}\left(x^{*}\right)
\end{aligned}
$$

We now show that the origin is GAS of (9).

It is easy to see $\phi_{i}(0)=0, \forall i=1,2, \cdots, n$. and

$$
\begin{aligned}
& \|\Phi(z(\cdot))\|^{2} \leq k z^{T}(\cdot) \Phi(z(\cdot)) \\
& \|\Psi(z(\cdot))\| \leq l\|\Phi(z(\cdot))\|
\end{aligned}
$$

Let us consider the Lyapunov function:

$$
\begin{aligned}
V(z(t))=\|z(t)\|^{2} & +2 \alpha \sum_{i=1}^{n} \int_{0}^{z_{i}} \phi_{i}(s) d s \\
& +\frac{1}{l^{2}}(\alpha+\beta) \sum_{i=1}^{n} \int_{t-\tau}^{t} \psi_{i}^{2}\left(z_{i}(\xi)\right) d \xi
\end{aligned}
$$

with $\alpha, \beta>0$ being chosen appropriately later on. We first point out that $V(z(\cdot))$ is positive except at the origin, and it is radially unbounded in the sense that $V(z(t)) \rightarrow \infty$ as $\|z(t)\| \rightarrow \infty$. Next, evaluating the time derivative of $V(z)$ along the trajectories of (9), we obtain

$$
\begin{aligned}
& \dot{V}(z(t))=2 z^{T}(t) \dot{z}(t)+2 \alpha \Phi^{T}(z(t)) \dot{z}(t) \\
& \quad+\frac{1}{l^{2}}(\alpha+\beta)\left(\|\Psi(z(t))\|^{2}-\|\Psi(z(t-\tau))\|^{2}\right) \\
& =-2 z^{T}(t)\left(g\left(z(t)+x^{*}\right)-g\left(x^{*}\right)\right) \\
& \quad+2 z^{T}(t) A \Phi(z(t))+2 z^{T}(t) A^{\tau} \Psi(z(t-\tau)) \\
& \quad-2 \alpha \Phi^{T}(z(t))\left(g\left(z(t)+x^{*}\right)\right. \\
& \left.\quad-g\left(x^{*}\right)\right)+2 \alpha \Phi^{T}(z(t)) A \Phi(z(t)) \\
& \quad+2 \alpha \Phi^{T}(z(t)) A^{\tau} \Psi(z(t-\tau)) \\
& +\frac{1}{l^{2}}(\alpha+\beta)\left(\|\Psi(z(t))\|^{2}-\|\Psi(z(t-\tau))\|^{2}\right)
\end{aligned}
$$

The Lebourg theorem for Lipshcitz functions means that

$$
g\left(z(t)+x^{*}\right)-g\left(x^{*}\right)=D z(t)
$$

for some $D \in \cup_{y \in\left[x^{*}, x^{*}+z(t)\right]} \partial g(y)$

From the definition of $g$, matrix $D$ is diagonal, and we denote $D=\operatorname{diag}\left(d_{1}, d_{2}, \cdots, d_{n}\right)$. It is obvious to see $d_{i} \geq m$ for $i=1,2, \cdots, n$. We then have

$$
\begin{gathered}
z^{T}(t)\left(g\left(z(t)+x^{*}\right)-g\left(x^{*}\right)\right) \\
=\sum_{i=1}^{n} d_{i} z_{i}^{2}(t) \geq m\|z(t)\|^{2} \\
\Phi^{T}(z(t))\left(g\left(z(t)+x^{*}\right)-g\left(x^{*}\right)\right) \\
=\sum_{i=1}^{n} d_{i} \phi_{i}\left(z_{i}(t)\right) z_{i}(t) \\
\geq m z^{T}(t) \Phi(z(t)) \geq \frac{m}{k}\|\Phi(z(t))\|^{2}
\end{gathered}
$$

Putting those inequalities into (11) and using (10), we have

$$
\begin{aligned}
& \dot{V}(z(t)) \leq-2 m\|z(t)\|^{2}+2 z^{T}(t) A \Phi(z(t)) \\
& +2 z^{T}(t) A^{\tau} \Psi(z(t-\tau))-2 \alpha \frac{m}{k}\|\Phi(z(t))\|^{2} \\
& +2 \alpha \Phi^{T}(z(t)) A \Phi(z(t))+2 \alpha \Phi^{T}(z(t)) A^{\tau} \Psi(z(t-\tau)) \\
& \quad+\frac{1}{l^{2}}(\alpha+\beta)\left(\|\Psi(z(t))\|^{2}-\|\Psi(z(t-\tau))\|^{2}\right)
\end{aligned}
$$

Noticing that

$$
\begin{aligned}
& -m\|z(t)\|^{2}+2 z^{T}(t) A \Phi(z(t)) \\
& =-\| \sqrt{m} z(t)-\frac{1}{\sqrt{m}} A \Phi\left(z(t) \|^{2}\right. \\
& \quad+\frac{1}{m} \Phi^{T}(z(t)) A^{T} A \Phi(z(t)) \\
& -m\|z(t)\|^{2}+2 z^{T}(t) A^{\tau} \Psi(z(t-\tau)) \\
& =-\| \sqrt{m} z(t)-\frac{1}{\sqrt{m}} A^{\tau} \Psi\left(z(t-\tau) \|^{2}\right. \\
& +\frac{1}{m} \Psi^{T}(z(t-\tau))\left(A^{\tau}\right)^{T} A^{\tau} \Psi(z(t-\tau)) \\
& 2 \alpha \Phi^{T}(z(t)) A^{\tau} \Psi(z(t-\tau))+\frac{1}{l^{2}} \alpha\|\Psi(z(t-\tau))\|^{2} \\
& =-\alpha\left\|\frac{1}{l} \Psi(z(t-\tau))-l\left(A^{\tau}\right)^{T} \Phi(z(t))\right\|^{2} \\
& \quad+\alpha l^{2} \Phi^{T}(z(t)) A^{\tau}\left(A^{\tau}\right)^{T} \Phi(z(t))
\end{aligned}
$$

Rearranging terms in (19) and using above inequalities, 
we obtain

$$
\begin{aligned}
& \dot{V}(z(t)) \leq \frac{1}{m} \Phi^{T}(z(t)) A^{T} A \Phi(z(t)) \\
& +\frac{1}{m} \Psi^{T}(z(t-\tau))\left(A^{\tau}\right)^{T} A^{\tau} \Psi(z(t-\tau)) \\
& -2 \alpha \frac{m}{k}\|\Phi(z(t))\|^{2}+2 \alpha \Phi^{T}(z(t)) A \Phi(z(t)) \\
& +\alpha l^{2} \Phi^{T}(z(t)) A^{\tau}\left(A^{\tau}\right)^{T} \Phi(z(t))+\frac{1}{l^{2}} \alpha\|\Psi(z(t))\|^{2} \\
& +\frac{1}{l^{2}} \beta\|\Psi(z(t))\|^{2}-\frac{1}{l^{2}} \beta\|\Psi(z(t-\tau))\|^{2} \\
& \leq \frac{1}{m} \lambda_{\max }\left(A^{T} A\right)\|\Phi(z(t))\|^{2}+\frac{1}{m} \lambda_{\max }\left(\left(A^{\tau}\right)^{T} A^{\tau}\right) . \\
& \quad\|\Psi(z(t-\tau))\|^{2}-2 \alpha \frac{m}{k}\|\Phi(z(t))\|^{2} \\
& \quad+2 \alpha \Phi^{T}(z(t)) A \Phi(z(t)) \\
& +\alpha l^{2} \Phi^{T}(z(t)) A^{\tau}\left(A^{\tau}\right)^{T} \Phi(z(t))+\frac{1}{l^{2}} \alpha\|\Psi(z(t))\|^{2} \\
& \quad+\frac{1}{l^{2}} \beta\|\Psi(z(t))\|^{2}-\frac{1}{l^{2}} \beta\|\Psi(z(t-\tau))\|^{2}
\end{aligned}
$$

Let $\beta=\left(I^{2} / m\right) \lambda_{\max }\left(\left(A^{\tau}\right)^{T} A^{\tau}\right)$, we have

$$
\begin{aligned}
& \dot{V}(z(t)) \leq \frac{1}{m}\left(\lambda_{\max }\left(A^{T} A\right)\right. \\
& \left.\quad+l^{2} \lambda_{\max }\left(\left(A^{\tau}\right)^{T} A^{\tau}\right)\right)\|\Phi(z(t))\|^{2} \\
& -2 \alpha \frac{m}{k}\|\Phi(z(t))\|^{2}+2 \alpha \Phi^{T}(z(t)) A \Phi(z(t)) \\
& +\alpha l^{2} \Phi^{T}(z(t)) A^{\tau}\left(A^{\tau}\right)^{T} \Phi(z(t))+\frac{1}{l^{2}} \alpha\|\Psi(z(t))\|^{2} \\
& +2 \alpha \Phi^{T}(z(t))\left(A^{\tau}\right)^{T} \Psi(z(t)) \\
& -2 \alpha \Phi^{T}(z(t))\left(A^{\tau}\right)^{T} \Psi(z(t)) 2 \alpha \Phi^{T}(z(t)) A \Phi(z(t)) \\
& +\alpha l^{2} \Phi^{T}(z(t)) A^{\tau}\left(A^{\tau}\right)^{T} \cdot \Phi(z(t))+\frac{1}{l^{2}} \alpha\|\Psi(z(t))\|^{2} \\
& +\frac{1}{l^{2}} \beta\|\Psi(z(t))\|^{2}-\frac{1}{l^{2}} \beta\|\Psi(z(t-\tau))\|^{2}
\end{aligned}
$$

Using the fact that

$$
\begin{aligned}
& \alpha l^{2} \Phi^{T}(z(t)) A^{\tau}\left(A^{\tau}\right)^{T} \Phi(z(t)) \\
& \quad-2 \alpha \Phi^{T}(z(t))\left(A^{\tau}\right)^{T} \Psi(z(t)) \\
& \begin{array}{c}
=\alpha\left(l^{2} \Phi^{T}(z(t)) A^{\tau}\left(A^{\tau}\right)^{T} \Phi(z(t))\right. \\
\left.-2 \Phi^{T}(z(t))\left(A^{\tau}\right)^{T} \Psi(z(t))\right)
\end{array} \\
& \leq \alpha\left\|l\left(A^{\tau}\right)^{T} \Phi(z(t))-\Phi(z(t))\right\|^{2}-\frac{1}{l^{2}} \alpha\|\Psi(z(t))\|^{2} \\
& \leq \alpha\left\|l A^{\tau}-I\right\|^{2} \| \Phi\left(z(t)\left\|^{2}-\frac{1}{l^{2}} \alpha\right\| \Psi(z(t)) \|^{2}\right.
\end{aligned}
$$

We have from (14) that

$$
\begin{aligned}
& \dot{V}(z(t)) \leq \frac{1}{m}\left(\lambda_{\max }\left(A^{\tau} A\right)+l^{2} \lambda_{\max }\left(\left(A^{\tau}\right)^{T} A^{\tau}\right)\right) . \\
& \|\Phi(z(t))\|^{2}-2 \alpha \frac{m}{k}\|\Phi(z(t))\|^{2}-\alpha\left(\left\|l A^{\tau}-I\right\|^{2} .\right. \\
& \| \Phi\left(z(t)\left\|^{2}-\frac{1}{l^{2}} \alpha\right\| \Psi(z(t))\left\|^{2}+\frac{1}{l^{2}} \alpha\right\| \Psi(z(t)) \|^{2}\right. \\
& +2 \alpha \Phi^{T}(z(t))\left(A+A^{\tau}+l A+l A^{\tau}\right) \Phi(z(t)) \\
& \leq \frac{1}{m}\left(\lambda_{\max }\left(A^{\tau} A\right)+l^{2} \lambda_{\max }\left(\left(A^{\tau}\right)^{T} A^{\tau}\right)\right)\|\Phi(z(t))\|^{2} \\
& \quad+2 \alpha \frac{m}{k}\|\Phi(z(t))\|^{2}+2 \mu_{2}\left(A+l A^{\tau}\right)\|\Phi(z(t))\|^{2} \\
& \quad+\alpha\left\|l A^{\tau}-I\right\|^{2} \| \Phi\left(z(t) \|^{2}\right. \\
& \leq \frac{1}{m}\left(\lambda_{\max }\left(A^{\tau} A\right)+l^{2} \lambda_{\max }\left(\left(A^{\tau}\right)^{T} A^{\tau}\right)\right)\|\Phi(z(t))\|^{2} \\
& -\alpha\left(2 \frac{m}{k}-2 \mu_{2}\left(A+l A^{\tau}\right)-\left\|l A^{\tau}-I\right\| \|^{2}\right)\|\Phi(z(t))\|^{2}
\end{aligned}
$$

Now we consider the following three cases.

1) $\Phi(z(t)) \neq 0$ and $z(t) \neq 0$.It then follows from (15) and $2 \mu_{2}\left(A+I A^{\tau}\right)+\left\|I A^{\tau}-I\right\|^{2}<2(m / k)$ that the choice

$$
\alpha>\frac{\lambda_{\max }\left(A^{T} A\right)+l^{2} \lambda_{\max }\left(\left(A^{\tau}\right)^{T} A^{\tau}\right)}{m\left(\frac{2 m}{k}-2 \mu_{2}\left(A+l A^{\tau}\right)-\left\|l A^{\tau}-I\right\|^{2}\right)}
$$

ensures that $\dot{V}(z(t))$ is negative.

2) $\Phi(z(t))=0$ but $z(t) \neq 0$. Then it follows from (13) that

$$
\begin{gathered}
\dot{V}(z(t)) \leq-2 m\|z(t)\|^{2}+2 z^{T}(t) A^{\tau} \Psi(z(t-\tau)) \\
+\frac{1}{l^{2}}(\alpha+\beta)\left(\|\Psi(z(t))\|^{2}-\|\Psi(z(t-\tau))\|^{2}\right) \\
=-m\|z(t)\|^{2}+2 z^{T}(t) A^{\tau} \Psi(z(t-\tau))-m\|z(t)\|^{2} \\
\quad+\frac{1}{l^{2}}(\alpha+\beta)\left(\|\Psi(z(t))\|^{2}-\|\Psi(z(t-\tau))\|^{2}\right) \\
\leq \frac{1}{m} \Psi^{T}(z(t-\tau))\left(A^{\tau}\right)^{T} A^{\tau} \Psi(z(t-\tau)) \\
+\frac{1}{l^{2}}(\alpha+\beta)\left(-\|\Psi(z(t-\tau))\|^{2}\right)+(\alpha+\beta)\|\Phi(z(t))\|^{2} \\
\left.\leq-\left(\frac{1}{l^{2}}(\alpha+\beta)-\frac{1}{m} \lambda_{\max }\left(\left(A^{\tau}\right)^{T} A^{\tau}\right)\right)\right)\|\Psi(z(t-\tau))\|^{2}
\end{gathered}
$$

We recall that $\beta=\left(l^{2} / m\right) \lambda_{\max }\left(\left(A^{\tau}\right)^{T} A^{\tau}\right)$, which obviously implies $\dot{V}(z(t))<0$ for this case.

3) $z(t)=0$,clearly, $\Phi(z(t))=0$ due the fact (10). In this case, $\dot{V}(z(t))$ is given by 


$$
\begin{aligned}
& \dot{V}(z(t))= \\
& \quad \frac{1}{l^{2}}(\alpha+\beta)\left(\|\Psi(z(t))\|^{2}-\|\Psi(z(t-\tau))\|^{2}\right) \\
& \leq(\alpha+\beta)\|\Phi(z(t))\|^{2}-\frac{1}{l^{2}}(\alpha+\beta)\|\Psi(z(t-\tau))\|^{2} \\
& \leq-\frac{1}{l^{2}}(\alpha+\beta)\|\Psi(z(t-\tau))\|^{2}
\end{aligned}
$$

Hence, $\dot{V}(z(t))$ is negative if $\Psi(z(t-\tau)) \neq 0$ and $\dot{V}(z(t))=0$ if and only if it happens in the last case where

$$
z(t)=\Phi(z(t))=\Psi(z(t-\tau))=0
$$

We recall that $V(z(t))$ is radially unbounded. According to [12] or [13] that the origin of (9) or equivalently the equilibrium point $x^{*}$ of (1) is GAS.

The sufficient conditions (8) for DCNNs are direct consequence of the general result proved above.

Theorem 4: In addition to assumptions (A1), (A2) and (A3), we assume that all the activation $f_{i}$ and $h_{i}$ are nondecreasing. Suppose that there exists a positive diagonal matrix $P=\operatorname{diag}\left(p_{1}, p_{2}, \cdots, p_{n}\right)$ such that the matrix

$$
P A+A^{T} P+l^{2}\left(P A^{\tau}\right)\left(P A^{\tau}\right)^{T}-2 M K^{-1} P+I
$$

is negative definite. Then for each $u \in \mathfrak{R}^{n}$, (1) has a unique equilibrium point which is GAS.

Proof: We first prove the existence and uniqueness of the equilibrium point by using Theorem 1 .

Let $C=\operatorname{diag}\left(c_{1}, c_{2}, \cdots, c_{n}\right)$ be a diagonal matrix with $c_{i} \geq m_{i}$ for all $i=1,2, \cdots, n$. Since both $K$ and $P$ are diagonally positive, the negative definiteness of the matrix in (16) implies that the matrix

$$
P A+A^{T} P+l^{2}\left(P A^{\tau}\right)\left(P A^{\tau}\right)^{T}-2 C K^{-1} P+I
$$

is also negative definite. By using positive semi definiteness of the matrix $X X^{T}+I-\left(X+X^{T}\right)$, we have that the matrix

$$
P A+A^{T} P+l^{2}\left(P A^{\tau}+\left(P A^{\tau}\right)^{T}\right)-2 C K^{-1} P
$$

is negative definite. In the other word, the matrix $-\left(A+l A^{\tau}-C K^{-1}\right)$ is Lyapunov diagonally stable under (16). Consequently, it is a $P$ matrix. Then it follows from [9] that for any diagonal matrix $D$ such that $0 \leq D \leq K, \operatorname{det}\left(C-\left(A+l A^{\tau}\right) D\right) \neq 0$; that is, the matrix $C-\left(A+l A^{\tau}\right) D$ is nonsingular for the above choice of $C$ and $D$. This fact in turn means that any element in $V$ is nonsingular. Now the existence and uniqueness of the equilibrium point follows from Theorem 1.

Next we prove the GAS of the equilibrium point, say $x^{*}$, of (1). Through the transformation as in the proof of Theorem 3, we consider the GAS of the system (9) at the origin. Define a Lyapunov function as [10] follows:

$$
\begin{aligned}
V(z(t))= & \|z(t)\|^{2}+2 \alpha \sum_{i=1}^{n} p_{i} \int_{0}^{z_{i}} \varphi_{i}(s) d s \\
& +(\alpha+\beta) \sum_{i=1}^{n} \int_{t-\tau}^{t} \varphi_{i}^{2}\left(z_{i}(\xi)\right) d \xi
\end{aligned}
$$

with $\alpha, \beta>0$,being selected later on. It is easy to see that $V(z(\cdot))$ is positive except at the origin and it is radially unbounded. Evaluating its time derivative along the trajectory of (9), we obtain that

$$
\begin{aligned}
\dot{V}(z(t)) & =-2 z^{T}(t)\left(g\left(z(t)+x^{*}\right)-g\left(x^{*}\right)\right) \\
+ & 2 z^{T}(t) A \Phi(z(t))+2 z^{T}(t) A^{\tau} \Psi(z(t)) \\
& -2 \alpha \Phi^{T}(z(t)) P\left(g\left(z(t)+x^{*}\right)\right. \\
& +2 \alpha \Phi^{T}(z(t)) P A \Phi(z(t)) \\
& +2 \alpha \Phi^{T}(z(t)) P A^{\tau} \Psi(z(t-\tau)) \\
& +\frac{1}{l^{2}}(\alpha+\beta)\left(\|\Psi(z(t))\|^{2}-\|\Psi(z(t-\tau))\|^{2}\right)
\end{aligned}
$$

Using the Lebourg theorem of (12) and as of deriving (13), we obtain

$$
\begin{aligned}
& \dot{V}(z(t))=-2 m\|z(t)\|^{2}+2 z^{T}(t) A \Phi(z(t)) \\
&+2 z^{T}(t) A^{\tau} \Psi(z(t))-2 \alpha \Phi^{T}(z(t)) P M K^{-1} \Phi(z(t)) \\
&+2 \alpha \Phi^{T}(z(t)) P A \Phi(z(t)) \\
&+2 \alpha \Phi^{T}(z(t)) P A^{\tau} \Psi(z(t-\tau)) \\
&+\frac{1}{l^{2}}(\alpha+\beta)\left(\|\Psi(z(t))\|^{2}-\|\Psi(z(t-\tau))\|^{2}\right)
\end{aligned}
$$

Let $\beta=\left(l^{2} / m\right) \lambda_{\max }\left(\left(A^{\tau}\right)^{T} A^{\tau}\right)$, we obtain similarly as of (22) that

$$
\begin{aligned}
& V(z(t))=\frac{1}{m}\left(\lambda_{\max }\left((A)^{T} A+l^{2} \lambda_{\max }\left(\left(A^{\tau}\right)^{T} A^{\tau}\right)\right)\right. \\
& \quad \times\|z(t)\|^{2}+2 \alpha \Phi^{T}(z(t)) P A \Phi(z(t)) \\
& \quad-\alpha \Phi^{T}(z(t))\left(2 P M K^{-1}-I\right) \Phi(z(t)) \\
& \quad+\alpha l^{2} \Phi^{T}(z(t)) P A^{\tau}\left(P A^{\tau}\right)^{T} \Phi(z(t)) \\
& =\frac{1}{m}\left(\lambda _ { \operatorname { m a x } } \left((A)^{T} A+l^{2} \lambda_{\max }\left(\left(A^{\tau}\right)^{T} A^{\tau}\right) \times\|z(t)\|^{2}\right.\right. \\
& +\alpha \Phi^{T}(z(t))\left(P A+A^{T} P+l^{2} P A^{\tau}\left(P A^{\tau}\right)^{T}\right. \\
& \left.\quad-2 P M K^{-1}+I\right) \Phi(z(t))
\end{aligned}
$$

Since the matrix in (16) is assumed to be negative definite, we can prove that the origin is GAS of (9) by following the very similar way of 1)-3) in the last part of the proof of Theorem 3 . This accomplishes our proof. 


\section{Illustrative Examples}

Example 1: Consider the following model:

$$
\left\{\begin{array}{c}
\dot{x_{1}}(t)=-g\left(x_{1}(t)\right)+A f\left(x_{1}(t)\right)+A^{\tau} h\left(x_{1}(t-\tau)\right)+u_{1} \\
\dot{x_{2}}(t)=-g\left(x_{2}(t)\right)+A f\left(x_{2}(t)\right)+A^{\tau} h\left(x_{2}(t-\tau)\right)+u_{2} \\
\dot{x} \\
\dot{x}_{3}(t)=-g\left(x_{3}(t)\right)+A f\left(x_{3}(t)\right)+A^{\tau} h\left(x_{3}(t-\tau)\right)+u_{3}
\end{array}\right.
$$

where $g(x)=0.3 x, f(x)=0.2 x, h(x)=0.1(x-\sin x)$, obviously, $g(x), f(x), h(x)$ satisfied the assumption (A1), (A2) and (A3), we obtain

$$
\begin{aligned}
& m=0.3, \quad k=0.2, \quad l=0.1 \\
& \text { Let } \quad A=\left[\begin{array}{ccc}
0.2 & -0.1 & 0 \\
0.1 & 0.3 & -0.2 \\
-0.2 & 0.1 & 0.2
\end{array}\right] \\
& A^{\tau}=\left[\begin{array}{ccc}
0.1 & 1 & 0.2 \\
-0.1 & 0.2 & 0.1 \\
0.2 & -0.1 & 0.4
\end{array}\right] \quad u=\left[\begin{array}{l}
0.1 \\
0.2 \\
0.1
\end{array}\right]
\end{aligned}
$$

It is easy to check that the model satisfied the condition of Theorem 3. Using the Matlab, we have made graphics of the solution as the time in the system with initial conditions [-0.5 $0.6-0.8]$ and the delay $\tau=0$, as follow: (Figure 1)

From the figure, we can easily see the system has a unique equilibrium point, and is GAS.

Example 2: In order to demonstrate the validity of our criterion of the Theorem 4, we consider a delayed neural network in (5) with parameters as

$$
\begin{gathered}
A=\left[\begin{array}{cc}
0.2 & 0.1 \\
-0.1 & 0.2
\end{array}\right] A^{\tau}=\left[\begin{array}{ll}
0.4 & 0.1 \\
0.5 & 0.5
\end{array}\right] \quad u=\left[\begin{array}{c}
1 \\
0.5
\end{array}\right] \\
g(x)=\left(\begin{array}{l}
0.2 x_{1} \\
0.1 x_{2}
\end{array}\right) \quad f(x)=\left(\begin{array}{l}
0.4 x_{1} \\
0.4 x_{2}
\end{array}\right) \\
h_{j}\left(x_{j}\right)=\left(\begin{array}{c}
0.2\left(x_{1}-\sin x_{1}\right) \\
0.2\left(x_{2}-\sin x_{2}\right)
\end{array}\right)
\end{gathered}
$$

obviously, (A1), (A2) and (A3) hold.

$$
M=\left[\begin{array}{cc}
0.2 & 0 \\
0 & 0.1
\end{array}\right] \quad K=\left[\begin{array}{cc}
0.4 & 0 \\
0 & 0.4
\end{array}\right] \quad l=0.1
$$

Using the Matlab LMI toolbox, we prove that the ma-

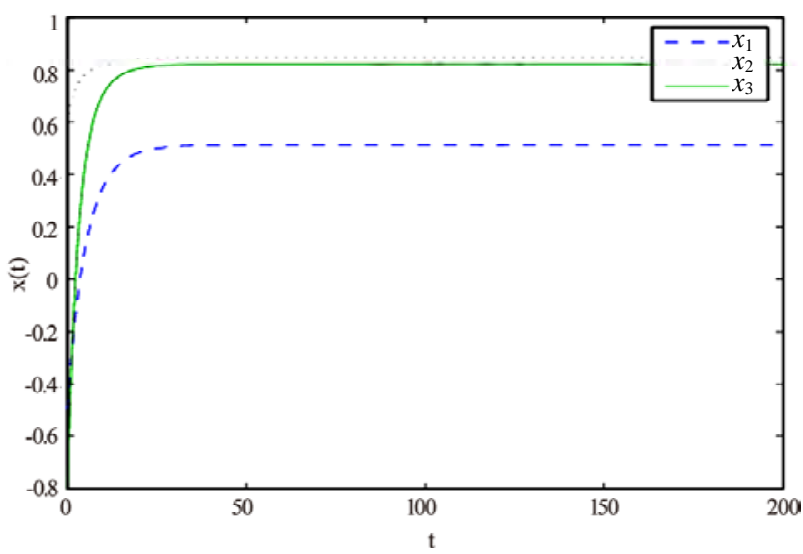

Figure 1. State trajectories of $x_{1}, x_{2}, x_{3}$.

trix inequality (17) is feasible. Also, we obtain the matrix

$$
P=\left[\begin{array}{cc}
1.169 & 0.000 \\
0.000 & 1.2765
\end{array}\right]
$$

\section{Conclusions}

In this paper, we present new conditions for the existence, uniqueness, and GAS of the equilibrium point of DNNs. Our study is based on a thorough nonsmooth analysis on functions defining DNNs. The general Theorem 1 on existence and uniqueness of the equilibrium point is proved easy to apply. This general result, allows us to study sufficient conditions for GAS in which the spectral properties of the matrix $\left(A+l A^{\tau}\right)$ play an important role. Advantages of our results are illustrated by examples and also given a graph of the GAS. It would be very interesting to see how our approach can be used to study conditions which do not enjoy symmetric properties.

\section{References}

[1] S. Arik, "An improved global stability result for delayed cellular neural networks,” IEEE Transactions on Circuits and Systems Part I, Vol. 49, pp. 1211-1214, 2002.

[2] T. L. Liao and F. C. Wang, "Global stability for cellular neural networks with time delay,” IEEE Transactions on Neural Networks, Vol. 11, pp. 1481-1484, 2000.

[3] Q. Zhang, X. Wei, and J. Xu, "Global asymptotic stability analysis of neural networks with time-varying delays," Neural Processing Letters, Vol. 21, pp. 61-71, 2005.

[4] Q. Zhang, X. Wei, and J. Xu, "Global asymptotic stability of cellular neural networks with infinite delay,” Neural Network World, Vol. 15, pp. 579-589, 2005.

[5] Q. Zhang, X. Wei, and J. Xu, "Stability analysis for cellular neural networks with variable delays," Chaos, Solitons Fractals, Vol. 28, pp. 331-336, 2006. 
[6] R. A. Horn and C. A. Johnson, "Matrix analysis,” U. K. Cambridge University Press, Cambridge, 1985.

[7] R. T. Rockafellar and R. J. B. Wets, "Variational analysis," Springer-Verlag, Berlin Heideberg, Germany, 1998.

[8] F. H. Clarke, "Optimization and nonsmooth analysis," Wiley, New York, 1983.

[9] B. H. Pourciau, "Hadamard theorem for locally Lipschitzian maps," Journal of Mathematical Analysis and Applications, Vol. 85, pp. 279-285, 1982.

[10] A. L. Dontchev, H.-D. Qi, and L. Qi, “Convergence of
Newton's method for convex best interpolation,” Numerical Mathematik, Vol. 87, pp. 435-456, 2001.

[11] L. Qi and J. Sun, “A nonsmooth version of Newton's method,” Mathematical Programming, Vol. 58, pp. 353367, 1993.

[12] H. K. Khalil, "Nonlinear systems," Macmillan, New York, 1988.

[13] R. M. Lewis and B. O. Anderson, "Intensitivity of a class of nonlinear comparetmental systems to the introduction of arbitrary time delays," IEEE Transactions on Circuits and Systems Part I, Vol. CAS-27, pp. 604-612, 1980. 